\title{
Bisphosphonate-associated atypical femur fracture in a 90-year-old Caucasian woman
}

\author{
KG Gopinath *, PK Shibu
}

Hong Kong Med J 2015;21:286.e2-3

DOI: $10.12809 / \mathrm{hkmj} 144384$

A 90-year-old Caucasian female was admitted to the hospital following a fall preceded by left thigh pain for 2 weeks in May 2013. Her medical history included postmenopausal osteoporosis, depression, ischaemic heart disease, and atrial fibrillation. She was on alendronate for 11 years, as well as aspirin, bisoprolol, digoxin, calcium, vitamin $\mathrm{D}$, frusemide, metformin, paracetamol, pantoprazole, and sertraline for around 14 years prior to hospital admission.

The Figure shows an atypical diaphyseal fracture of the femur commonly associated with long-term bisphosphonate therapy. These fractures usually occur in patients taking bisphosphonates for more than 5 years although it is known to occur with shorter duration of usage and in bisphosphonatenaive patients (10\%). ${ }^{1}$ This patient fulfilled all the ASBMR (American Society for Bone and Mineral Research) task force major and minor criteria for atypical fractures. ${ }^{2}$ The mandatory major criterion is fracture located along the femoral diaphysis from just distal to the lesser trochanter to just proximal to the supracondylar flare. In addition, at least four of five major features must be present: (1) The fracture is associated with minimal or no trauma, as in a fall from a standing height or less. (2) The fracture line originates at the lateral cortex and is substantially transverse in its orientation, although it may become oblique as it progresses medially across the femur. (3) Complete fractures extend through both cortices and may be associated with a medial spike; incomplete fractures involve only the lateral cortex. (4) The fracture is non-comminuted or minimally comminuted. (5) There is localised periosteal or endosteal thickening of the lateral cortex at the fracture site ("beaking" or "flaring"). The minor features not essential for diagnosis include: generalised increase in cortical thickness of the femoral diaphysis, unilateral or bilateral prodromal symptoms such as dull or aching pain in the groin or thigh, bilateral incomplete or complete femoral diaphysis fracture, and delayed fracture healing. Differential diagnoses in these patients include femoral fractures with subtrochanteric extension, pathological fractures associated with tumours, and periprosthetic fractures. $^{2}$

Management strategies include cessation of bisphosphonates, protected weight-bearing, prophylactic intramedullary rod insertion, and use of anabolic bone agents like teriparatide or strontium. ${ }^{3}$ It is unclear whether a drug holiday is useful to prevent these fractures. ${ }^{4}$ Our patient was treated with intramedullary nailing and commenced

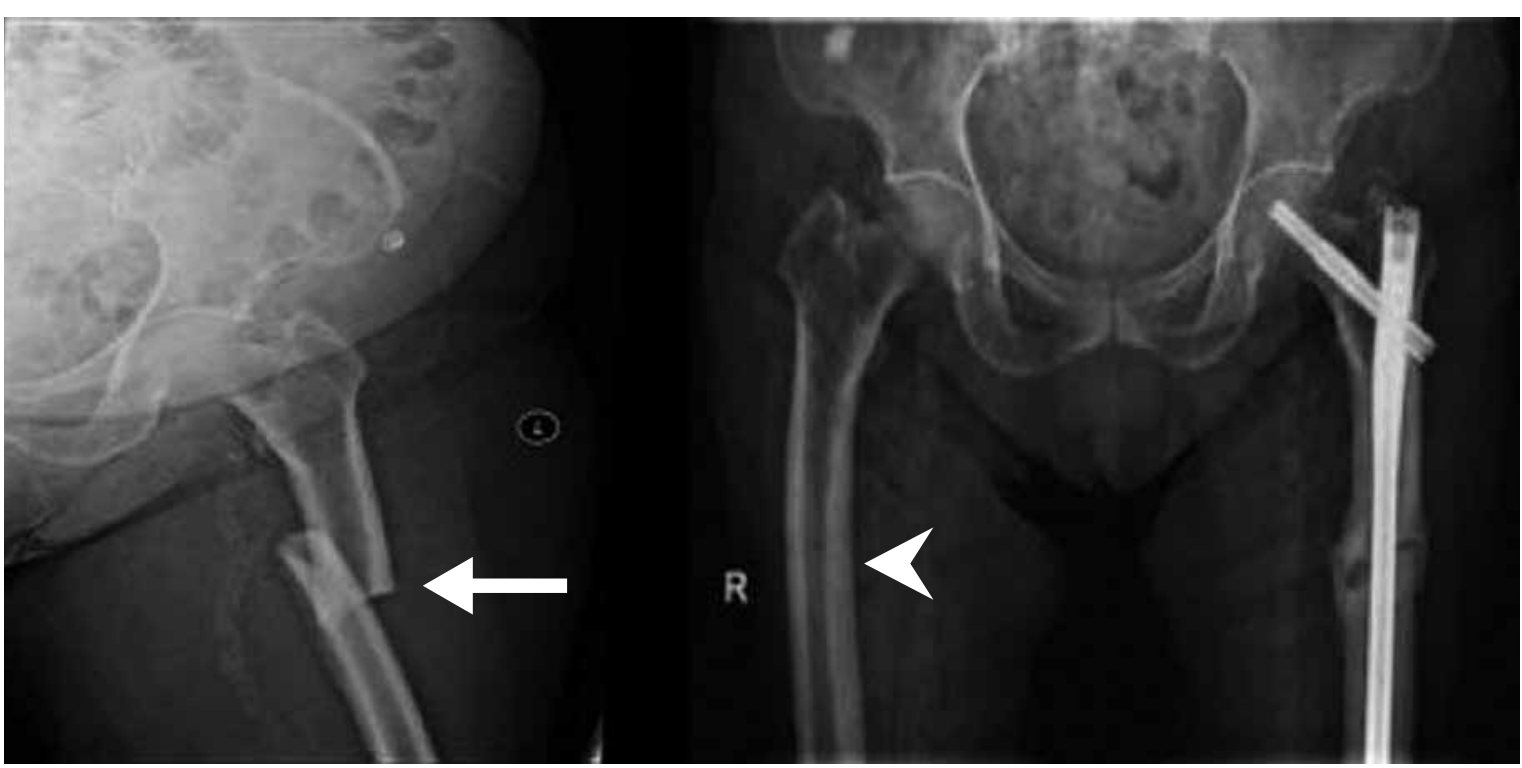

FIG. Complete transverse, non-comminuted diaphyseal fracture of the left femur (arrow) is shown. Cortical thickening associated with bowing (arrowhead) is noted in the right femur 
on strontium after cessation of bisphosphonates. Greater awareness of this condition would prevent misdiagnosis especially in frail older patients and facilitate proper management.

\section{Declaration}

Dr PK Shibu has received educational grants and honorarium from Novartis Pty Australia for Osteoporosis and Fracture Liaison related clinical research projects or lectures in the past and received honorarium from Amgen Ltd for presenting at educational meetings in the past.

KG Gopinath *, MD, FRACP

PK Shibu, FRACP, MRCP

Aged and Extended Care Services, The Queen Elizabeth Hospital, Woodville SA 5011, University of Adelaide, SA 5000, Australia
* Corresponding author: gops95@yahoo.com

\section{References}

1. Dell RM, Adams AL, Greene DF, et al. Incidence of atypical nontraumatic diaphyseal fractures of the femur. J Bone Miner Res 2012;27:2544-50.

2. Shane E, Burr D, Abrahamsen B, et al. Atypical subtrochanteric and diaphyseal femoral fractures: second report of a task force of the American Society for Bone and Mineral Research. J Bone Miner Res 2014;29:1-23.

3. Carvalho NN, Voss LA, Almeida MO, Salgado CL, Bandeira F. Atypical femoral fractures during prolonged use of bisphosphonates: short-term responses to strontium ranelate and teriparatide. J Clin Endocrinol Metab 2011;96:2675-80.

4. Diab DL, Watts NB. Bisphosphonate drug holiday: who, when and how long. Ther Adv Musculoskelet Dis 2013;5:107-11. 